ANNALES

POLONICI MATHEMATICI

LXXVIII.2 (2002)

\title{
Borel methods of summability and ergodic theorems
}

\author{
by RYSZARD JAJTE (Łódź)
}

\begin{abstract}
Passing from Cesàro means to Borel-type methods of summability we prove some ergodic theorem for operators (acting in a Banach space) with spectrum contained in $\mathbb{C} \backslash(1, \infty)$.
\end{abstract}

1. Introduction. Let $X$ be a Banach space. Denote by $B(X)$ the algebra of bounded linear operators acting in $X$. Take $u \in B(X)$. If the Cesàro averages

$$
n^{-1} \sum_{k=0}^{n-1} u^{k}
$$

converge, say, weakly then the spectrum of $u$ is necessarily contained in the unit disc $\{|z| \leq 1\}$. Passing from the Cesàro means to the Borel-type methods of summability [4], [5] one can extend the ergodic theorems to the case of operators $u$ with the spectrum $\sigma(u)$ contained in the Mittag-Leffler star for $z \mapsto(1-z)^{-1}$, i.e. with $\sigma(u) \subset \mathbb{C} \backslash(1, \infty)$. A discussion of such possibilities is the main goal of the paper.

Let us begin with some notation and definitions. For $\alpha>0$ and a sequence $x=\left(\xi_{n}\right)$ of numbers (or vectors), put

$$
B_{\alpha}(t, x)=\alpha e^{-t} \sum_{n=0}^{\infty} \frac{t^{n \alpha}}{\Gamma(n \alpha+1)} \xi_{n}, \quad t>0 .
$$

The function $B_{\alpha}(t, x)$ is called the $B_{\alpha}$-transform of the sequence $x=\left(\xi_{n}\right)$. If $\lim _{t \rightarrow \infty} B_{\alpha}(t, x)=\xi$, then we say that $\left(\xi_{n}\right)$ is summable to $\xi$ by the method $B_{\alpha}$, and write $\xi_{n} \rightarrow \xi\left(B_{\alpha}\right)$, or $B_{\alpha}-\lim _{n \rightarrow \infty} \xi_{n}=\xi$. The family of methods $\left\{B_{\alpha}: \alpha>0\right\}$ is consistent, i.e. for every $\alpha^{\prime}, \alpha^{\prime \prime}>0, B_{\alpha^{\prime}}-\lim \xi_{n}=\xi$ and $B_{\alpha^{\prime \prime}}-\lim \xi_{n}=\eta$ implies $\xi=\eta$ (cf. [5]). For our purposes it will be enough to take $\alpha=2^{-k}, k=0,1, \ldots$, so in what follows we consider only the family $\mathcal{B}=\left\{B_{2^{-k}}: k=0,1, \ldots\right\}$. By the consistency just mentioned the family $\mathcal{B}$

2000 Mathematics Subject Classification: 47A35, 60F15.

Key words and phrases: summability method, ergodic theorem. 
may be treated as a $\mathcal{B}$-method of summability: a sequence $x$ is $\mathcal{B}$-summable when it is $B_{2^{-k}}$-summable for some $k \in \mathbb{N}$. Borel methods of summability are right-translative, i.e. the $B_{\alpha}$-summability of $x=\left(\xi_{0}, \xi_{1}, \ldots\right)$ implies the $B_{\alpha}$-summability of $x^{-}=\left(0, \xi_{0}, \xi_{1}, \ldots\right)$. Notice that the $B_{\alpha}$-method is not left-translative, i.e. the $B_{\alpha}$-summability of $x$ does not imply, in general, the $B_{\alpha}$-summability of $x^{+}=\left(\xi_{1}, \xi_{2}, \ldots\right)$ (cf. [5]).

Before formulating the main results let us start with the following lemma.

2. Lemma. Fix $\alpha=2^{-k}$ and $0<d<1$. Put

$$
D_{\alpha, d}=\{z \in \mathbb{C}: \operatorname{Re} z \leq 0\} \cup\left\{z \in \mathbb{C}: \operatorname{Re} z^{1 / \alpha} \leq 1-d\right\} .
$$

Let $\Delta$ be a bounded Borel subset of $D_{\alpha, d}$. Here and elsewhere let $\zeta=\left(z^{n}\right)$. Then, for $t>0$ and $z \in \Delta$,

$$
\left|B_{\alpha}(t, \zeta)\right| \leq C e^{-d t / 2},
$$

for some constant $C$ depending only on $\Delta$.

Proof. A crucial point in the proof is a suitable representation of the Mittag-Leffler function

$$
E_{\alpha}(w)=\sum_{n=0}^{\infty} \frac{w^{n}}{\Gamma(n \alpha+1)}, \quad \alpha>0, \quad \text { for } w=t^{\alpha} z .
$$

We follow Włodarski [5]. Let us remark that, for a fixed $\alpha=2^{-k}$, the function

$$
f(t)=E_{\alpha}\left(t^{\alpha} z\right)=\sum_{n=0}^{\infty} \frac{t^{n \alpha}}{\Gamma(n \alpha+1)} z^{n}
$$

(as a function of $t>0$ ) satisfies the differential equation

$$
f^{\prime}(t)=g(t)+z^{2^{k}} f(t) \quad \text { with } \quad g(t)=\sum_{\nu=1}^{2^{k}-1} \frac{t^{\nu 2^{-k}-1}}{\Gamma\left(\nu 2^{-k}\right)} z^{\nu} .
$$

This is easy to check. Consequently, we have

$$
f(t)=\exp \left(z^{2^{k}} t\right)\left[1+\int_{0}^{t} \exp \left(-z^{2^{k}} u\right) \sum_{\nu=1}^{2^{k}-1} \frac{u^{\nu 2^{-k}-1}}{\Gamma\left(\nu 2^{-k}\right)} z^{\nu} d u\right] .
$$

The substitution $z^{2^{k}}=v t$ leads to the formula

$$
\begin{aligned}
& \sum_{n=0}^{\infty} \frac{t^{n 2^{-k}}}{\Gamma\left(n 2^{-k}+1\right)} z^{n} \\
& \quad=\exp \left(t z^{2^{k}}\right)\left[1+\sum_{\nu=1}^{2^{k}-1} \alpha_{\nu}^{(k)}(z) \int_{\left[0, z^{2^{k}} t\right]} \frac{e^{-v} v^{\nu 2^{-k}-1}}{\Gamma\left(\nu 2^{-k}\right)} d v\right],
\end{aligned}
$$


where

$$
\alpha_{\nu}^{(k)}(z)=\frac{z^{\nu}}{\left[x^{2^{k} \nu}\right]^{1 / 2^{k}}}=e^{i \theta_{\nu}^{(k)}(z)} .
$$

The functions $\alpha_{\nu}^{(k)}(z)$ are determined by fixing the rational power $w \mapsto$ $w^{1 / 2^{k}}$ as taking its values in the angle $\left\{z=r e^{i \theta}: r \geq 0,-\pi / 2^{k-1}<\theta \leq\right.$ $\left.\pi / 2^{k-1}\right\}$. In particular, $\alpha_{1}^{(1)}(z)=-1$ for $\operatorname{Re} z<0$, and $\alpha_{\nu}^{(k)}(1)=1$ for $1 \leq \nu \leq 2^{k}-1, k=1,2, \ldots$

For $z=1$, the formula (4) gives

$$
\sum_{n=0}^{\infty} \frac{t^{n 2^{-k}}}{\Gamma\left(n 2^{-k}+1\right)}=e^{t}\left[1+\sum_{\nu=1}^{2^{k}-1} \frac{1}{\Gamma\left(\nu 2^{-k}\right)} \int_{[0, t]} e^{-u} u^{\nu 2^{-k}-1} d u\right]
$$

(cf. [5], p. 144).

Put $Q=\left\{z \in \mathbb{C}: \operatorname{Re} z^{2^{k}} \leq 1-d\right\}$.

For $\operatorname{Re} z<1, t>1$ and $\beta>-1$, we have the inequality

$$
\left|e^{t(z-1)} \int_{[0, z t]} u^{\beta} e^{-u} d u\right| \leq C|z t|^{\beta+1} \max \left(e^{-t}, e^{-t(1-\operatorname{Re} z)}\right) .
$$

We omit a rather standard proof.

Let $z \in \Delta \cap Q$, where $\Delta$ is a fixed bounded set. Then by (4) and (6), we get $(2)$.

Now assume that Re $z \leq 0$. Then, clearly,

$$
B_{1}(t, \zeta)=e^{-t(1-z)}
$$

so $\left|B_{1}(t, \zeta)\right| \leq e^{-t}$.

Consider the following transformation $W$ :

$$
W(f)(t)=\frac{e^{-t}}{2 \sqrt{\pi t}} \int_{0}^{\infty} \exp \left(-\frac{u^{2}}{4 t}+u\right) f(u) d u
$$

defined for continuous functions $f:(0, \infty) \rightarrow \mathbb{R}$ (cf. [5], p. 140). The transformation $W$ is regular in the sense that $\lim _{n \rightarrow \infty} f(u)=\beta$ implies $\lim _{t \rightarrow \infty} W(f)(t)=\beta$. Moreover, we have

$$
W\left(B_{2^{-k}}(\cdot, x)\right)(t)=B_{2^{-(k+1)}}(t), \quad t>0,
$$

Applying to both sides of (7) the $k$ th iteration of the transformation $W$ defined in (8) and taking into account the positivity of $W$ and (9) we easily get

$$
\left|B_{2^{-k}}(t, \zeta)\right| \leq C e^{-t} \text { for } \operatorname{Re} z \leq 0
$$

As an easy consequence of Lemma 2, we get the following result. 
3. Theorem (Uniform ergodic theorem). Let $u \in B(X)$ with $\sigma(u) \subset$ $\mathbb{C} \backslash(1, \infty)$. If $1 \notin \sigma(u)$ then there exists a $k \in \mathbb{N}$ such that

$$
B_{2^{-k}}-\lim _{n \rightarrow \infty} u^{n}=0 \quad \text { in the uniform operator topology. }
$$

If $1 \in \sigma(u) \subset \mathbb{C} \backslash(1, \infty)$ and 1 is a pole of $u$ of order one, then

$$
B_{2^{-k}-} \lim _{n \rightarrow \infty} u^{n}=\mathbb{E}\{1\} \quad \text { uniformly, }
$$

where $\mathbb{E}\{1\}$ denotes the spectral projection of $u$ at $\{1\}$.

Proof. Suppose $1 \notin \sigma(u)$. Since $\sigma(u)$ is compact and $\sigma(u) \subset \mathbb{C} \backslash[1, \infty)$, there exist $0<d<1$ and $k \in \mathbb{N}$ such that $\sigma(u) \subset D_{2^{-k}, d}=\{\operatorname{Re} z \leq 0\}$ $\cup\left\{\operatorname{Re} z^{2^{k}} \leq 1-d\right\}$. Let $R(\cdot, u)$ be the resolvent of $u$ and, for $x=\left(u^{n}\right)$, $\zeta=\left(z^{n}\right)$, let

$$
B_{2^{-k}}(t, x)=\frac{1}{2 \pi i} \int_{K} B_{2^{-k}}(t, \zeta) R(z, u) d z
$$

be a representation of the Borel transform $B_{2^{-k}}(t, x)$ as a Cauchy integral, i.e. $K$ is the (oriented) boundary of an open set $V \supset \sigma(u) ; K$ consists of a finite number of rectifiable Jordan curves (cf. [3], p. 568). By Lemma 2 we easily get

$$
\left\|B_{2^{-k}}(t, x)\right\| \leq C e^{-d t / 2}, \quad t>1 .
$$

Now, let $1 \in \sigma(u) \subset \mathbb{C} \backslash(1, \infty)$. Then, putting

$$
f_{t}(u)=B_{2^{-k}}(t, x), \quad x=\left(u^{n}\right),
$$

we can write

$$
f_{t}(u)=f_{t}(u) \mathbb{E}(\sigma(u) \backslash\{1\})+B_{2^{-k}}(t, \mathbf{1}) \mathbb{E}\{1\},
$$

with $\mathbf{1}=(1,1, \ldots)$, where, for a spectral set $A$ of $u, \mathbb{E}(A)$ denotes the corresponding projection operator (cf. [3], p. 573). To conclude the proof it is enough to pass with $t$ to infinity.

Taking discrete Borel methods, i.e. considering the transforms $B_{\alpha}(m, x)$ only for $m=1,2, \ldots$, we can easily prove the following theorem.

4. Theorem (Individual ergodic theorem). Let $X=\mathbb{L}_{p}(\mu), p \geq 1$, and let $u \in B(X)$ with $\sigma(u) \subset \mathbb{C} \backslash(1, \infty)$. If $1 \notin \sigma(u)$ then there exists a $k \in \mathbb{N}$ such that

$$
B_{2^{-k}-} \lim _{n \rightarrow \infty} u^{n} f=0 \quad \mu \text {-almost everywhere, for every } f \in X .
$$

If $1 \in \sigma(u) \subset \mathbb{C} \backslash(1, \infty)$ and 1 is a pole of $u$ of order one then, for every $f \in X$,

$$
B_{2^{-k}-} \lim _{n \rightarrow \infty} u^{n} f=\mathbb{E}\{1\} f \quad \mu \text {-almost everywhere. }
$$


Proof. The proof can be obtained as an easy modification of the previous argument. Namely, using the above estimates we get easily

$$
\sum_{m=1}^{\infty}\left\|B_{2^{-k}}(m, x) f\right\|_{p}^{p}<\infty,
$$

for every $f \in X$. The rest is trivial.

In the case $1 \in \sigma(u) \subset \mathbb{C} \backslash(1, \infty)$ and when 1 is not a simple pole one cannot expect the results as clear as the above theorems. The asymptotic behaviour of $u$ heavily depends on its spectral properties near the value 1 . The sequence $\left(z^{n}\right)$ with $z$ close to 1 is rather slowly divergent and Borel summability methods are efficient for rapidly divergent sequences (cf. [4]). It is worth noting here that for a sequence $\left(X_{n}\right)$ of independent identically distributed random variables the $\operatorname{limit} B_{1}-\lim X_{n}=\mathcal{E} X_{1}$ (expectation of $X_{1}$ ) exists almost everywhere if and only if $\mathcal{E}\left(X_{1}^{2}\right)<\infty$, so in the classical context of the Strong Law of Large Numbers, the Borel methods are less efficient than the Cesàro means (cf. [1], [2]).

Let $X$ be again an arbitrary Banach space. For $u \in B(X)$, we say that $\left(u^{n}\right)$ is strongly $B_{\alpha}$-summable to $P$ when $B_{\alpha}-\lim _{n \rightarrow \infty} u^{n} \xi=P \xi$ for every $\xi \in$ $X$. By the right-translativity of $B_{\alpha}$, we then also have $B_{\alpha}-\lim u^{n-1} \xi=P \xi$. By the continuity of $u$, we get $u P \xi=P \xi$. Consequently, $B_{\alpha}-\lim u^{n+1} \xi=P \xi$ (left-translativity of $B_{\alpha}$ for sequences of the form $\left(u^{n} \xi\right)$ ), and also $P^{2}=P$, $u P=P u$.

For $x=\left(u^{n}\right)_{n=0}^{\infty}$, let $x^{+}=\left(u^{n+1}\right)_{n=0}^{\infty}$.

5. Theorem (Mean ergodic theorem). Let $u \in B(X)$, where $X$ is a Banach space. Then the sequence $x=\left(u^{n}\right)_{n=0}^{\infty}$ is strongly $B_{\alpha}$-summable to a projection $Q$ if and only if the following conditions are satisfied:

(i) $\sup _{0<t<\infty}\left\|B_{\alpha}(t, x)\right\|<\infty$,

(ii) $B_{\alpha}\left(t, x^{+}-x\right) \rightarrow 0$ strongly as $t \rightarrow \infty$,

(iii) the family $\left\{B_{\alpha}(t, x): t>0\right\}$ is weakly sequentially compact.

Proof. Necessity. (i) is a consequence of the Banach-Steinhaus theorem. (ii) follows from the translativity of $B_{\alpha}$ for the sequence $\left(u^{n} \xi\right)$. (iii) is obvious.

Sufficiency. Put

$$
X_{0}=\{\xi \in X: u \xi=\xi\}, \quad X_{1}=\{u \xi-\xi: \xi \in X\}^{-} .
$$

Obviously, $B_{\alpha}-\lim u^{n} \xi=\xi=Q \xi$ for $\xi \in X_{0}$. Put $Y=\{u \xi-\xi: \xi \in X\}$. If $\eta \in Y$ then $\eta=u \xi-x$ for some $\xi \in X$, and we have, for $x=\left(u^{n}\right)$,

$$
\alpha e^{-t} \sum_{n=0}^{\infty} \frac{t^{n \alpha}}{\Gamma(n \alpha+1)} u^{n} \eta=B_{\alpha}\left(t, x^{+}-x\right) \rightarrow 0
$$

strongly as $t \rightarrow \infty$, by (ii). 
It is enough to show that $X=X_{0}+X_{1}$, because in this case the proof can be completed by a standard approximation. Fix $\xi \in X$. By (iii), we find a vector $\bar{\xi}$ such that

$$
\bar{\xi}=w-\lim _{k \rightarrow \infty} B_{\alpha}\left(t_{k}, x\right) \xi,
$$

for some $t_{k} \nearrow \infty$ (here $w$-lim denotes the weak limit).

We have

$$
\begin{aligned}
u \bar{\xi} & =w-\lim _{k \rightarrow \infty} u\left(B_{\alpha}\left(t_{k}, x\right) \xi\right) \\
& =w-\lim _{k \rightarrow \infty} B_{\alpha}\left(t_{k}, x^{+}-x\right) \xi+w-\lim _{k \rightarrow \infty} B_{\alpha}\left(t_{k}, x\right) \xi=\bar{\xi},
\end{aligned}
$$

by (ii). We have just proved that $\bar{\xi} \in X_{0}$, and we shall show that $\xi-\bar{\xi}$ $\in X_{1}$. By the Hahn-Banach theorem it is enough to check that, for every $\phi \in X^{*}$ which disappears on $X_{1}$, we have $\phi(\xi-\bar{\xi})=0$. But if $\phi=0$ on $X_{1}$ then, in particular, $\phi(u \xi)=\phi(\xi)$ for every $\xi \in X$, so $\phi(\xi)=\phi\left(u^{n} \xi\right)$, $n=1,2, \ldots$ Consequently,

$$
\phi\left(B_{\alpha}\left(t_{k}, x\right) \xi\right)=\alpha e^{-t_{k}} \sum_{n=0}^{\infty} \frac{t_{k}^{n \alpha}}{\Gamma(n \alpha+1)} \phi\left(u^{n} \xi\right)=\phi(\xi) B_{\alpha}\left(t_{k}, \mathbf{1}\right) .
$$

Passing with $k$ to infinity we get $\phi(\bar{\xi})=\phi(\xi)$, i.e. $\phi(\xi-\bar{\xi})=0$.

\section{References}

[1] N. H. Bingham, Summability methods and dependent strong laws, in: Progr. in Probab. Statist. 11, Birkhäuser, 1986, 291-300.

[2] N. H. Bingham and M. Maejima, Summability methods and almost sure convergence, Z. Wahrsch. Verw. Gebiete 68 (1985), 383-392.

[3] N. Dunford and J. T. Schwartz, Linear Operators, Part I: General Theory, Interscience, New York, 1964.

[4] G. W. Hardy, Divergent Series, Oxford Univ. Press, 1956.

[5] L. Włodarski, Sur les méthodes continues de limitation du type de Borel, Ann. Polon. Math. 4 (1958), 137-174.

Faculty of Mathematics

University of Łódź

Banacha 22

90-238 Łódź, Poland

E-mail: rjajte@math.uni.lodz.pl 